\title{
Regularities of Deformation Microstructures in Ferritic Stainless Steels during Large Strain Cold Working
}

\author{
Andrey BELYAKOV, ${ }^{1)}$ Kaneaki TSUZAKI ${ }^{1,2)}$ and Yuuji KIMURA ${ }^{11}$ \\ 1) Structural Metals Center, National Institute for Materials Science, 1-2-1, Sengen, Tsukuba, Ibaraki $305-0047$ Japan. \\ 2) Department of Materials Science and Engineering, Tokyo Institute of Technology, 4295, Nagatsuta, Midori, Yokohama 226- \\ 8502 Japan.
}

(Received on January 18, 2008; accepted on May 10, 2008)

\begin{abstract}
The recent studies on grain refinement in several $\mathrm{Cr}-\mathrm{Ni}$ ferritic stainless steels during large strain deformation at room temperature are critically reviewed. The paper is focussed on the mechanism of structural changes that is responsible for ultrafine grain development. It is concluded that the development of submicrocrystalline structures results from a kind of strain-induced continuous reaction; that is formation of deformation subboundaries and gradual increase in their misorientations up to typical values of ordinary grain boundaries. Following the rapid reduction at an early deformation, the transverse grain/subgrain size smoothly approaches its final value of the order of $0.1-0.2 \mu \mathrm{m}$, depending on alloying extent. The increase in average subboundary misorientation upon processing can be related to the strain as $\Delta \theta \sim K \varepsilon$, with $K$ of about $5^{\circ}$. Therefore, the average misorientation between deformation grains/subgrains is about $20^{\circ}$ after processing to total strain of 4 and the corresponding microstructure is composed of $50 \%$ of high-angle grain boundaries. The structural refinement of starting material can significantly accelerate the kinetics of ultrafine grain development during subsequent plastic working, while the processing method has a little effect on developing microstructures.
\end{abstract}

KEY WORDS: severe deformation; stainless steels; strain-induced grain boundaries; submicrocrystalline structures; continuous dynamic recrystallization.

\section{Introduction}

Ultrafine grained metals and alloys have been arousing a great interest among materials scientists and engineers. Such materials are believed to offer improved mechanical properties like a high strength that is combined with sufficient ductility, enhanced impact toughness, superplasticity at high strain rate, and many others, which were reported by various research groups. ${ }^{1-9)}$ One of processing methods for grain refinement in metallic materials is plastic deformation. Commonly, the development of new grained structure during plastic working is related to the phenomenon of dynamic recrystallization. The main characteristic of dynamic recrystallization taking place at elevated temperatures have been fairly clarified in a number of papers. ${ }^{10-14)}$ The dynamic grain size that can be obtained after completion of dynamic recrystallization decreases with a decrease in deformation temperature. Therefore, a considerable refinement of the microstructure can be achieved by plastic working at relatively low temperatures. However, the critical strain that is required for the initiation of dynamic recrystallization and, especially, for its completion increases significantly with decreasing the processing temperature.

Several specific processing methods such as torsion under high pressure, mechanical milling, equal channel angular pressing, and others have been developed to attain large strains during plastic deformation at low to moderate temperatures. ${ }^{3,5,15-19)}$ The novel methods of severe deformation allow treating almost all metallic materials. The grain size in structural materials can be reduced down to tens nanometers after processing by severe deformation. It is commonly discussed that the final microstructures result from continuous transformation of strain-induced subgrains into ultrafine grains due to gradual rise of angular misorientations among deformation substructures during deformation. ${ }^{3,20-23)}$ However, the sequence of structural changes, namely, the mechanism of submicrocrystalline or nanocrystalline structure evolution during large strain cold working is still unclear. The specific techniques mentioned above are tedious in use, require costly equipment and only enable the processing of relatively small samples. This makes the comprehensive structural investigations complicated. On the other hand, large strain deformation can be also achieved by some conventional metal-forming methods like drawing and multiple forging, which are simple in applications and based on standard tools. Recently, bar rolling followed by swaging and multiaxial forging were utilized as methods of severe deformation to process several stainless steels. ${ }^{24-26)}$ After sufficiently large strains, the submicrocrystalline structures were developed in the treated samples. The aim of the present paper is to summarize briefly our late results on severe deformations and to reveal the peculiarities of the structural mechanism responsible for the development of strain-induced submicrocrys- 
Table 1. Chemical composition and initial grain $\left(D_{0}\right)$ or subgrain $\left(d_{0}\right)$ size in stainless steels, which were used as the starting materials for large strain deformation by bar rolling/swaging and multiaxial forging. ${ }^{24-26)}$ Note that $D_{0}$ and $d_{0}$ are the values after hot rolling at $973 \mathrm{~K}$.

\begin{tabular}{lccccccc}
\hline Steels & $\mathrm{C}$ & $\mathrm{Mn}$ & $\mathrm{P}$ & $\mathrm{S}$ & $\mathrm{Cr}$ & $\mathrm{Ni}$ & $\mathrm{N}$ \\
\hline $\mathrm{Fe}-15 \mathrm{Cr}$, Ferrite, & 0.003 & 0.01 & 0.001 & 0.001 & 15.0 & - & 0.003 \\
$D_{0}=30 \mu \mathrm{m}$ & & & & & & & \\
\hline $\mathrm{Fe}-22 \mathrm{Cr}-3 \mathrm{Ni}$, Ferrite, & 0.002 & 0.01 & 0.005 & 0.001 & 21.99 & 3.12 & 0.002 \\
$D_{0}=700 \mu \mathrm{m}$ & & & & & & & \\
\hline $\mathrm{Fe}-18 \mathrm{Cr}-17 \mathrm{Ni}$, Martensite, & 0.008 & 0.01 & 0.004 & 0.001 & 18.05 & 6.99 & 0.001 \\
$d_{0}=230 \mathrm{~nm}$ & & & & & & & \\
\hline Fe $-27 \mathrm{Cr}-9 \mathrm{Ni}$, Ferrite/Austenite (3/2), & 0.017 & 0.01 & 0.01 & 0.001 & 26.8 & 9.03 & 0.002 \\
$d_{\mathrm{F}}=700 \mathrm{~nm}, d_{\mathrm{A}}=400 \mathrm{~nm}$ & & & & & & & \\
\hline
\end{tabular}

talline structures on the base of quantitative analysis of deformation microstructures that evolve at different strain levels.

\section{Experimental}

Various $\mathrm{Cr}-\mathrm{Ni}$ stainless steels were chosen as typical representatives of a wide grade of structural alloys. The chemical composition of starting materials is collected in Table 1 along with some microstructural parameters. The steels were vacuum melted and cast into $20 \mathrm{~kg}$ ingots followed by homogenization and hot rolling at $973 \mathrm{~K}$ to $21.3 \mathrm{~mm} \times 21.3 \mathrm{~mm}$ square bars. The processing details are explained elsewhere. ${ }^{24-26)}$ The main results were obtained by using unidirectional deformation. Namely, the large strain cold working was carried out by bar rolling to $7.8 \mathrm{~mm} \times 7.8 \mathrm{~mm}$ square bars followed by swaging from $\phi 7.0 \mathrm{~mm}$ to about $\phi 0.5 \mathrm{~mm}$. Therefore, the total strain of around 7 was attained. To study the effect of processing method on grain refinement under severe deformation, a portion of samples was subjected to multidirectional deformation by multiple forging with change of the loading direction through $90^{\circ}$ from pass to pass (i.e. $x$ to $y$ to $z$ to $x$ and so on). The specimens for multiple forging were machined in rectangular shape with starting dimensions of $15.0 \mathrm{~mm} \times 12.2 \mathrm{~mm} \times 10.0 \mathrm{~mm}$. The applied strain at each processing pass was 0.4 . The total strain of 7.2 was obtained after 18 sequential forging passes.

\section{Grain Refinement during Large Strain Cold Work- ing}

\subsection{Deformation Microstructures}

Representative micrographs of deformation microstructures that developed in an originally coarse grained ferritic stainless steel during bar rolling/swaging to various total strains are shown in Fig. 1. After cold rolling to conventional strain level, the strain-induced substructures are characterized by high density of dislocations, which are arranged in cell blocks separated by dense dislocation walls (Fig.

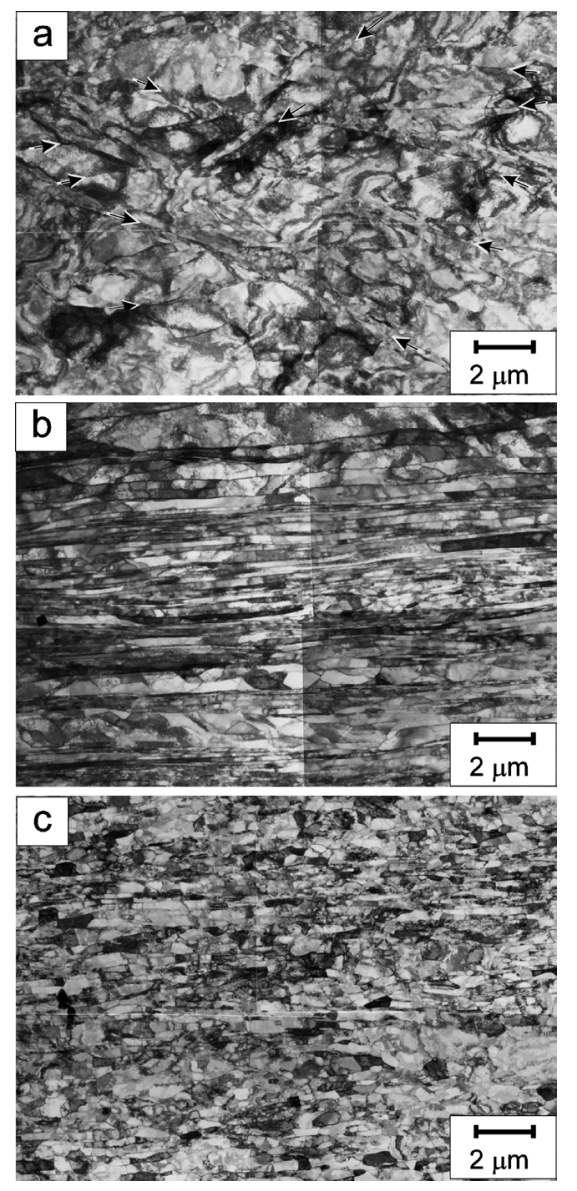

Fig. 1. Typical microstructures evolved in $\mathrm{Fe}-22 \mathrm{Cr}-3 \mathrm{Ni}$ after cold working by bar rolling/swaging to total strains of $\varepsilon=1.0$ (a), $\varepsilon=3.2$ (b), $\varepsilon=4.4$ (c). The deformation axis is horizontal in the figures.

1(a)). This is a typical feature of cold worked microstructure that was frequently observed in numerous studies on conventional plastic working. ${ }^{21,27-30)}$ It should be noted in Fig. 1(a) that some dense dislocation walls appear as long straight boundaries (some of those are indicated by arrows). They are rather sharp on the TEM images, comparing to the ordinary dislocation cell walls. Therefore, such subboundaries can be considered as geometrically necessary subboundaries subdividing the original large grains into microvolumes, the combination of operating slip systems within which is different. ${ }^{31,32)}$ The misorientation across such subboundaries is expected to rise rapidly due to accumulation of misfit dislocations that are resulted from the difference in dislocation slip in neighbor subgrains. ${ }^{33)}$

Further processing results in rearrangement of the dense dislocation walls, which become almost parallel to the deformation axis (Fig. 1(b)). In other words, the deformation substructures are composed by highly elongated subgrains at a level of intermediate strains of around 3. Such ribbonlike substructures are distinctive characteristics of unidirectional deformation to relatively large strains. ${ }^{27,34-36)}$ The spacing between these longitudinal subboundaries was discussed to decrease with subsequent working. However, the TEM observations of the deformation substructures that developed after severe deformation (Fig. 1(c)) suggest that the transverse subgrain size does not reduce remarkably in the range of very large strains. On the other hand, many dis- 

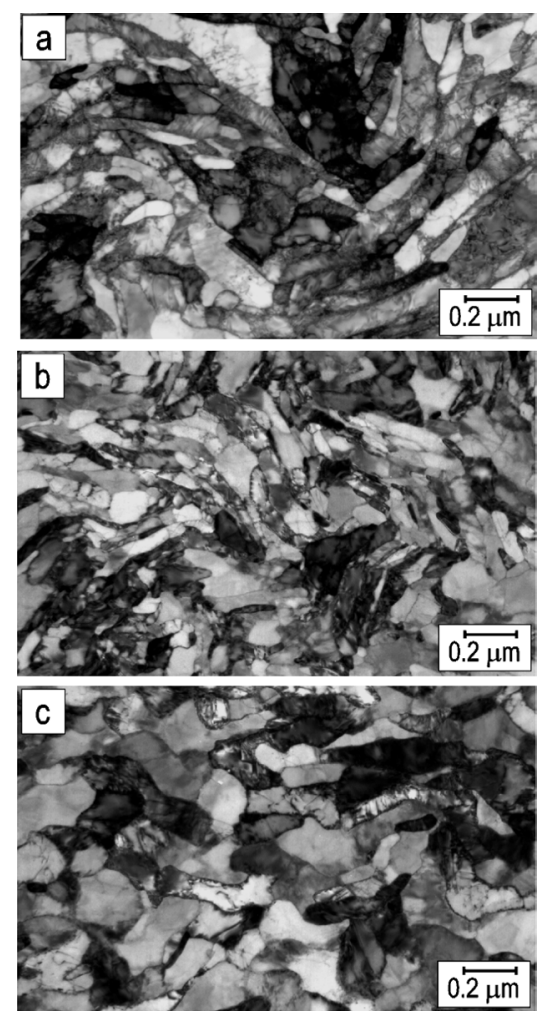

Fig. 2. Deformation microstructures in $\mathrm{Fe}-18 \mathrm{Cr}-7 \mathrm{Ni}$ after cold rolling/drawing to total strains of $\varepsilon=2.0$ (a), $\varepsilon=3.2$ (b), $\varepsilon=4.4$ (c); the transverse section view.

location subboundaries develop crosswise to the processing axis. The evolution of transverse subboundaries breaks down the ribbon-like microstructure into chains of slightly elongated subgrains, leading to the formation of submicrocrystalline structures in severely deformed samples.

The change in the shape of strain-induced grains/subgrains during large strain cold working can also be seen in the transverse sections of bar rolled/swaged samples. ${ }^{37)}$ At the level of conventional strains up to about 3 , the deformation microstructures look like ordinary ones that develop upon unidirectional deformation (Figs. 2(a) and 2(b)). Such microstructures are composed of curly grains/subgrains, which are bent around the deformation axis. The thickness of bent plate grains reduces with increasing the total strain. In contrast, almost equiaxed ultrafine grains/subgrains appear in the cross section of the samples after sufficiently large strains (Fig. 2(c)). It can be concluded that severe deformation is characterized by the evolution of more equiaxed (sub)structural elements than the deformation substructures at intermediate strain level.

The reduction of the transverse grain/subgrain size, which was measured crosswise to the direction of metal flow during rolling/swaging, is represented in Fig. 3 for various steels. ${ }^{24-26)}$ Early deformation is characterized by a rapid drop of the transverse (sub)grain size. This is consistent with a work hardening taking place right after beginning the plastic flow, when the dislocation density readily increases that leads to formation of cell substructure followed by the development of many dense dislocation walls subdividing original grains. ${ }^{27,34)}$ Then, the rate of reduction of the transverse (sub)grain size slows down; and the size of structural elements gradually approaches its minimal

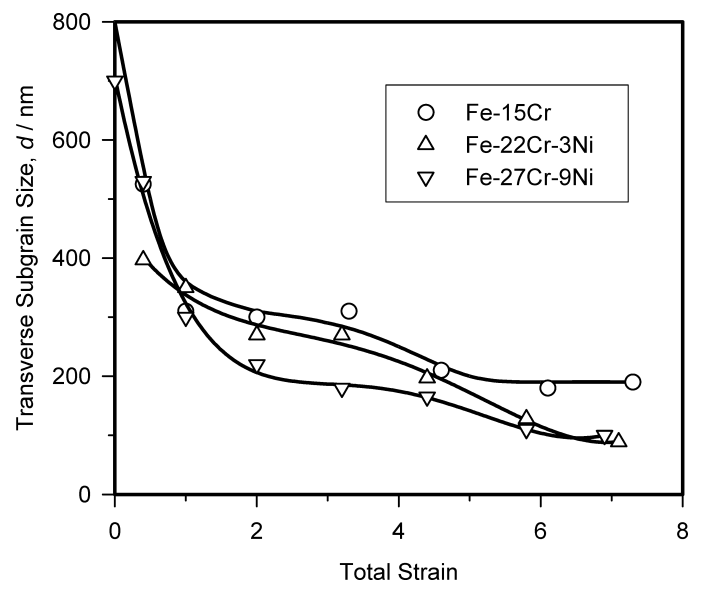

Fig. 3. Reduction of the transverse (sub)grain size during severe deformation by bar rolling/swaging.

value at saturation level of $100-200 \mathrm{~nm}$ that depends on alloying extent. In other words, the transverse grain/subgrain size does not vary with subsequent processing in the range of very large total strains.

\subsection{Evolution of Strain-induced Subboundaries}

The refinement of original microstructures during large strain cold working is accompanied by increase in misorientations between deformation subgrains (Fig. 4). The substructures consisting of cell blocks that developed at relatively small strains are characterized mainly by low-angle dislocation subboundaries (Fig. 4(a)). The dense dislocation walls with misorientations above $15^{\circ}$ are spaced far from each other and, therefore, rare in occurrence. Further processing to intermediate total strains results in significant increase of the number of the dense dislocation walls and rearranges the latter ones along the deformation axis. It is clearly seen in Fig. 4(b) that the development of ribbon-like microstructures attends a remarkable increase in the misorientations between deformation (sub)grains. Almost half of the dense dislocation walls among all the strain-induced (sub)boundaries is represented by high-angle grain boundaries. It should also be noted that an increase in misorientations between subgrains is accompanied with sharpening of deformation subboundaries. The strain-induced (sub)boundaries with relatively large misorientations appear as narrow plane defects and commonly look like ordinary grain boundaries. The fraction of strain-induced high-angle grain boundaries continuously increases upon subsequent deformation. Therefore, the following evolution of submicrocrystalline structures at large total strains is associated with a gradual transformation of deformation subgrains into ultrafine grains with high-angle boundaries. It is concluded that the submicrocrystalline structures that develop under severe deformation result from a kind of continuous dynamic recrystallization. The average misorientation between deformation (sub)grain and the fraction of strain-induced high-angle grain boundaries can be used as quantitative parameters to follow the kinetics of the process.

Effect of the total strain on the average (sub)grain boundary misorientation and the fraction of high-angle grain boundaries is shown in Fig. 5. The orientations of individual (sub)grains were obtained by the Kikuchi-line diffrac- 

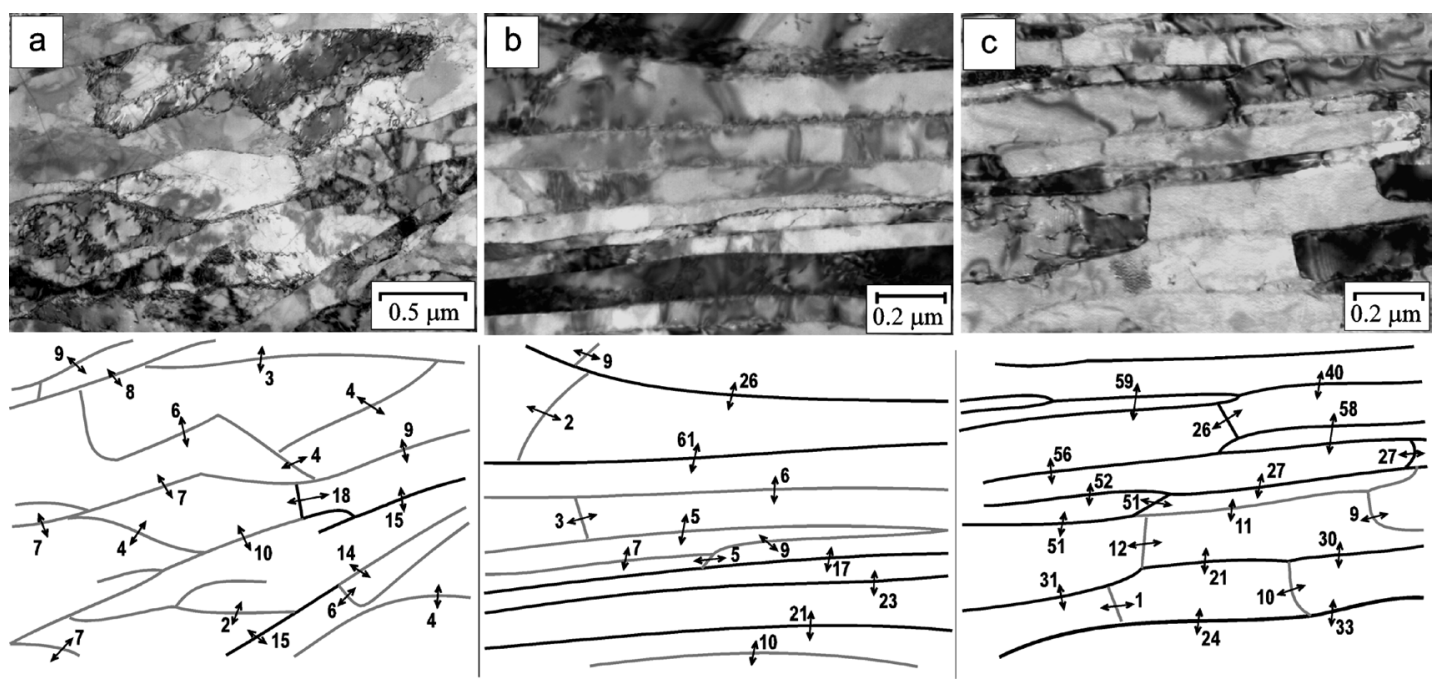

Fig. 4. Evolution of strain-induced (sub)boundaries in $\mathrm{Fe}-22 \mathrm{Cr}-3 \mathrm{Ni}$ steel during cold working to total strains of $\varepsilon=2.0$ (a), $\varepsilon=3.2$ (b), $\varepsilon=7.1$ (c); the numbers indicate the boundary misorientations in degrees.

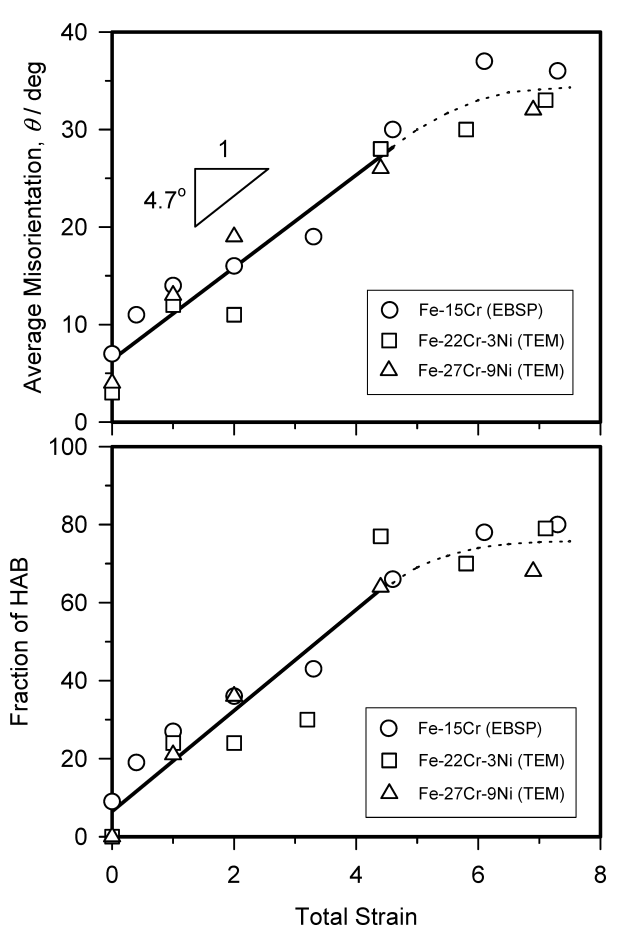

Fig. 5. Increase in misorientations between deformation grains/ subgrains upon cold rolling/swaging.

tion technique using transmission electron microscope $(\mathrm{TEM})^{24,25)}$ and an electron back scattering diffraction pattern (EBSP) analyzer. ${ }^{26)}$ Both the average misorienation and the fraction of high-angle boundaries gradually increase with deformation to total strains of about 4 and approach saturations at larger strains, where the average misorientation is around $35^{\circ}$ and the percentage of high-angle boundaries comprises about $75 \%$. Similar structural changes were frequently observed for continuous dynamic recrystallization in various materials. ${ }^{20,21,38-40)}$ It is interesting to note that in the range of the strains of $0<\varepsilon<4$, the both boundary parameters can be roughly approximated by a linear relationship with the total strain, i.e. $\theta \sim K \varepsilon$. For the average misorientation, the factor $K$ is about $5^{\circ}$ in Fig. 5. This value is almost the same with those of $4^{\circ}$ to $7^{\circ}$ obtained for con-

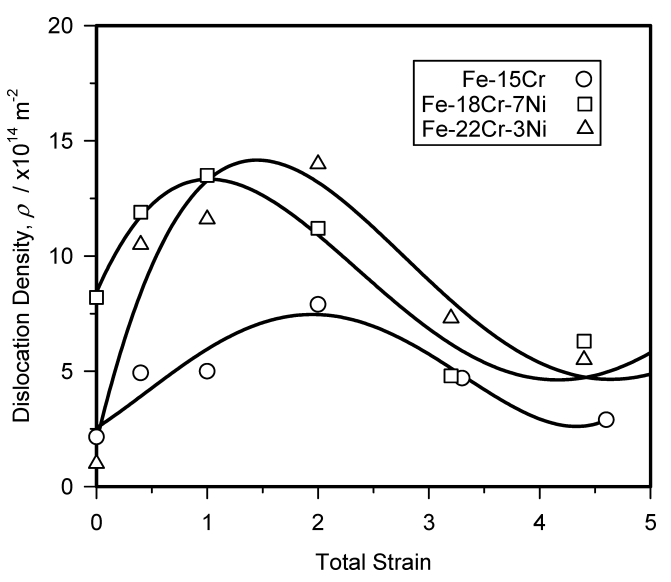

Fig. 6. Variation of dislocation density in grain/subgrain interiors during large strain processing

tinuous dynamic recrystallization in various metallic materials, which were deformed to large strains at relatively low temperatures. ${ }^{21)}$ Larger $K$ values were observed for processing at elevated temperatures, e.g. $K=12^{\circ}$ for cyclic hot rolling and annealing ${ }^{41)}$ and very large values of the misorientation rate up to $40^{\circ}$ for aluminum alloys during hot working in conditions of superplastic deformation. ${ }^{42}$ The linear relationship between the average misorientation and the strain can be explained with an assumption that the main contribution in the rise of misorientations is provided by the geometrically necessary subboundaries. The misorientation across such subboundaries depends on the density of grain boundary dislocations, which in turn is in direct proportion to the strain, ${ }^{43)}$ therefore, $\theta \sim \varepsilon$.

\subsection{Internal Stresses}

The submicrocrystalline structures developed by severe deformation involve a smaller number of internal dislocations compared to the deformation substructures after conventional strains (see Figs. 1 and 2). The variation of the dislocation density in the grain/subgrain interiors with total strain is represented in Fig. 6. ${ }^{24-26)}$ The number of internal dislocations increases to its maximum upon processing to total strains of about 1 to 2 . Then, the dislocation density 
smoothly decreases with following deformation. On the other hand, the submicrocrystalline materials subjected to severe deformation were shown bearing high residual stresses. ${ }^{20,24,44-46)}$ To study the origin of the internal stresses in the submicrocrystalline structures, some of the ultrafine grains without any internal dislocations were selected for more detailed examination.

An example of dislocation-free grains is displayed in Fig. $7{ }^{37)}$ The arrows indicate on microregions, where the orientations of the crystal lattice were precisely evaluated by the low-angle converged beam Kikuchi-line diffraction technique. The numbers indicate the local disorientations or the curvatures $(\psi)$ of the grain lattice in the diffraction plane between the neighboring microregions. The maximal residual stresses associated with these elastic distortions can be roughly derived as follows ${ }^{45)}: \tau / G=0.35 t \psi / l$, where $G$ is the shear modulus, $t$ is the specimen thickness, and $l$ is the spacing between the microregions. The level of the internal stresses of about $2 \times 10^{-3} \mathrm{G}$ can be averaged through the A-B-C-D-E in Fig. 7. Since these ultrafine grains are free of dislocations in their interiors, it is evident that such high internal stresses should be attributed to the strain-induced grain boundaries in submicrocrystalline structures obtained by large strain deformation. Also, the high internal stresses that evolve in submicrocrystalline structures after

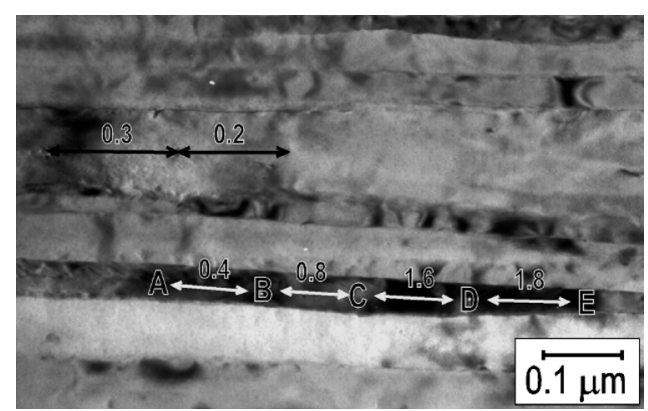

Fig. 7. Dislocation-free strain-induced (sub)grains in $\mathrm{Fe}-18 \mathrm{Cr}-$ $7 \mathrm{Ni}$ after severe deformation. The numbers indicate the local lattice curvatures between the pointed microregions in degrees. severe deformation are considered to be responsible for decreasing the density of internal dislocations after sufficiently large strains. ${ }^{20,46)}$

\section{Effect of Processing Method}

To study the effect of processing method on the development of submicrocrystalline structures due to continuous dynamic recrystallization under severe deformation, two quite different techniques, i.e. the unidirectional deformation by bar rolling/swaging and the multidirectional deformation by three-dimensional forging, were selected for cold-working of the same starting material. ${ }^{26)}$ Typical deformation structures that evolve upon the different deformations to various total strains are represented in Fig. 8. At relatively small total strains, the effect of processing method on structural changes is negligible. The deformation substructures consisting of dislocation cell blocks that evolve by unidirectional and multidirectional deformations are the same in appearance (Figs. 8(a) and 8(d)). The similar cold-worked substructures were frequently observed in many studies irrespective of used processing method. ${ }^{21,27-30)}$

As expected, remarkable difference in the deformation substructures is recorded for samples processed to relatively large (intermediate) strains (Figs. 8(b) and 8(e)). The unidirectional deformation causes the strain-induced subboundaries to become parallel to the processing axis, leading to ribbon-like substructures, which are composed by highly elongated (sub)grains. In contrast, the deformation substructures that develop under multidirectional forging consist of almost equiaxed grains/subgrains within the studied strain range. The subdivision of elongated (sub)grains by transverse strain-induced (sub)boundaries in the swaged samples after large total strains eliminates the structural differences introduced by unidirectional and multidirectional deformation (Figs. 8(c) and 8(f)).

In spite of certain differences in deformation structures evolved by different processing methods, the transverse grain/subgrain size can be represented by a unique function of the total strain and does not depend on the method of
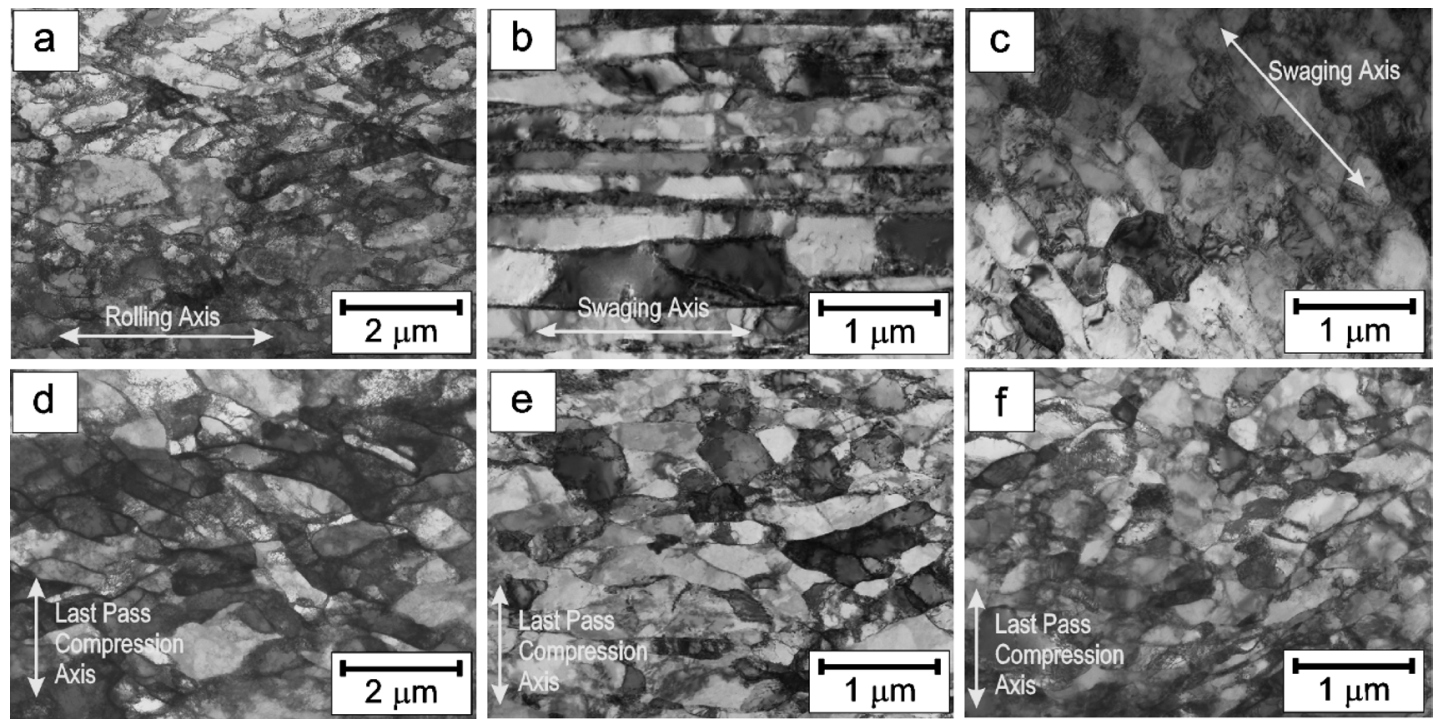

Fig. 8. Deformation microstructures in $\mathrm{Fe}-15 \mathrm{Cr}$ after unidirectional deformation to total strains of $\varepsilon=1.0$ (a), $\varepsilon=4.6$ (b), $\varepsilon=7.3$ (c); and those after multidirectional deformation to total strains of $\varepsilon=0.8$ (d), $\varepsilon=4.0$ (e), $\varepsilon=7.2$ (f). 


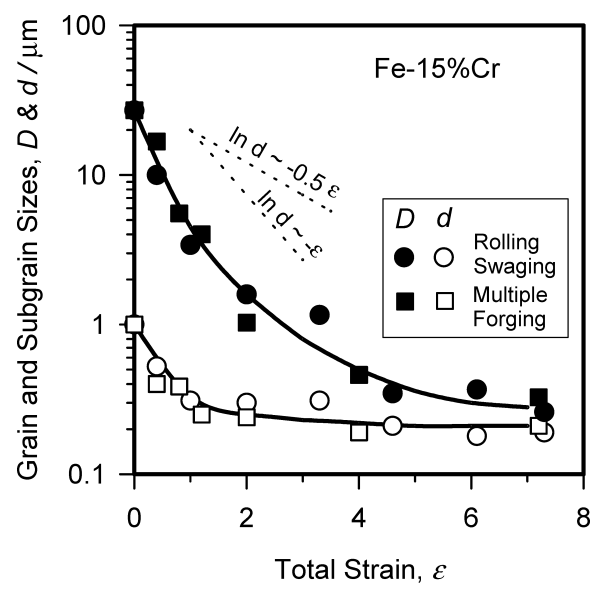

Fig. 9. Reduction of the transverse grain and subgrain sizes during unidirectional and multidirectional severe deformations.

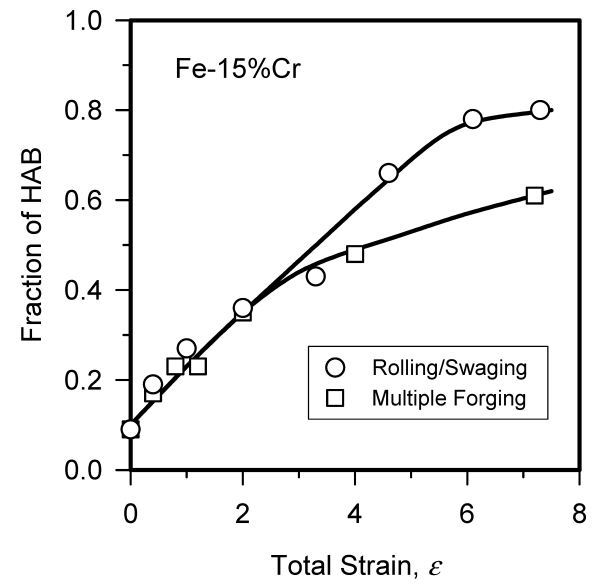

Fig. 10. Effect of processing method on the fraction of strain-induced high-angle grain boundaries.

cold working (Fig. 9). This suggests that the size of ultrafine grains that develop by continuous dynamic recrystallization during severe deformation can be reduced down to some limit, which depends on physical deformation conditions such as temperature and strain rate. This is similar to the phenomenon of discontinuous dynamic recrystallization during hot working, when the dynamic grain size can be expressed by a power law function of temperature compensated strain rate, ${ }^{10-14)}$ although responsible structural mechanisms seem to be different. In the case of continuous dynamic recrystallization, the final grain size approaches the size of dislocation subgrains or cells, which reaches a limit in the early stages of deformation and changes only slightly thereafter. $^{43}$ )

The kinetics of continuous dynamic recrystallization during unidirectional and multidirectional working can be seen in Fig. 10, which shows the increase in the fraction of strain-induced high-angle grain boundaries as a function of the total strain. Similar to the data presented in Fig. 5, the fraction of high-angle boundaries increases in a linear dependent way with straining to total strains of about 4 irrespective of the difference in the processing methods. Upon subsequent deformation to large strains, the fraction of high-angle boundaries tends to saturate at 0.8 and 0.6 for swaging and multiple forging, respectively. This difference

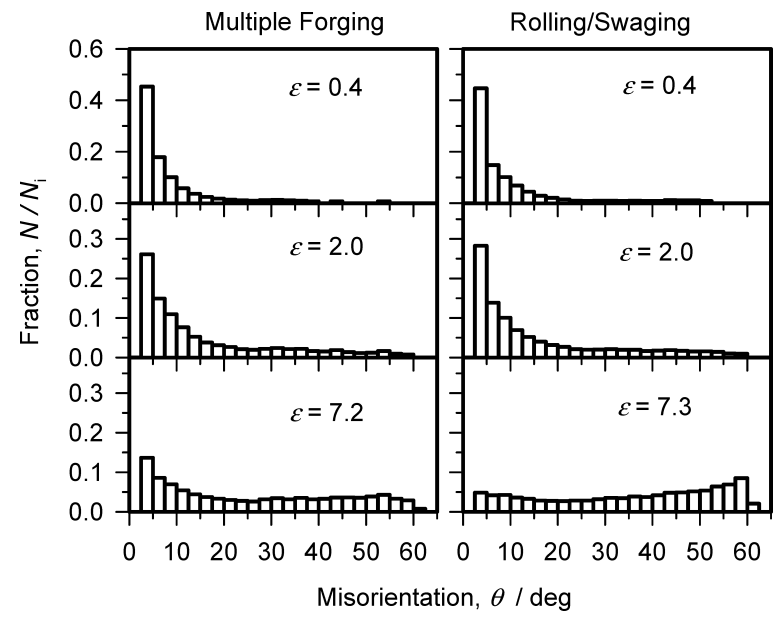

Fig. 11. Misorientation distributions for deformation (sub)boundaries in $\mathrm{Fe}-15 \mathrm{Cr}$ processed by multiple forging and bar rolling/swaging to various total strains.

in the fraction of high-angle boundaries at large total strains is associated with the specific distributions for (sub)boundary misorientations that develop during multidirectional and unidirectional working (Fig. 11). In the both cases, the peak against low-angle subboundaries gradually decreases during processing and the fraction of grain boundaries with misorientation above $15^{\circ}$ increases. However, the multidirectional deformation leads to a flat-type (sub)boundary distribution with near the same fractions of various misorientations, while the unidirectional rolling/swaging is characterised by a rather large fraction of $60^{\circ}$ grain boundary misorientations that appear at large strains. The latter may be connected with the strong $\langle 110\rangle$ fiber texture in the rolled/swaged samples, ${ }^{24-26)}$ because the misorientation between grains rotating about $\langle 110\rangle$ through $60-90^{\circ}$ is represented by an equivalent minimal misorientation about $60^{\circ}$.

\section{Effect of Initial Microstructure}

The fast development of strain-induced high-angle boundaries was shown to take place close to original grain boundaries. ${ }^{39)}$ Deformation subboundaries evolved near initial grain boundaries have higher misorientations, as compared with those in grain interiors, and can rapidly transform to high-angle ones due to some strain incompatibilities of adjacent grains. Therefore, the kinetics of grain refinement during large strain cold working is expected to be accelerated in materials with finer starting microstructures. This statement was clearly proved by using two stainless steels with the same alloying extent but different initial microstructures. $^{24)}$ The development of strain-induced ultrafine grained structure in the samples of $\mathrm{Fe}-18 \% \mathrm{Cr}-7 \% \mathrm{Ni}$ steel with initial martensitic microstructure was compared with the structural changes in coarse grained $\mathrm{Fe}-22 \% \mathrm{Cr}-$ $3 \% \mathrm{Ni}$ samples during the same processing method.

The kinetics of the development of high-angle grain boundaries upon processing is illustrated by Fig. 12, which represents the misorientation distributions for the deformation (sub)boundaries. The sharp peak against low-angle dislocation subboundaries in the samples with initial coarse grained microstructure slowly decreases and spreads out towards larger misorientations during processing. The charac- 


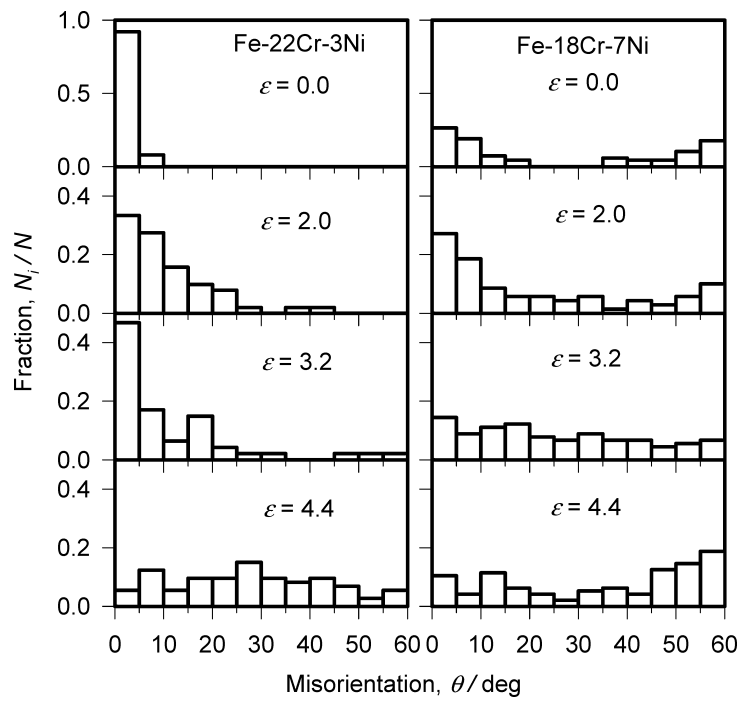

Fig. 12. Misorientation distributions for deformation (sub)boundaries in $\mathrm{Fe}-22 \mathrm{Cr}-3 \mathrm{Ni}$ and $\mathrm{Fe}-18 \mathrm{Cr}-7 \mathrm{Ni}$ processed by bar rolling/swaging to various total strains.

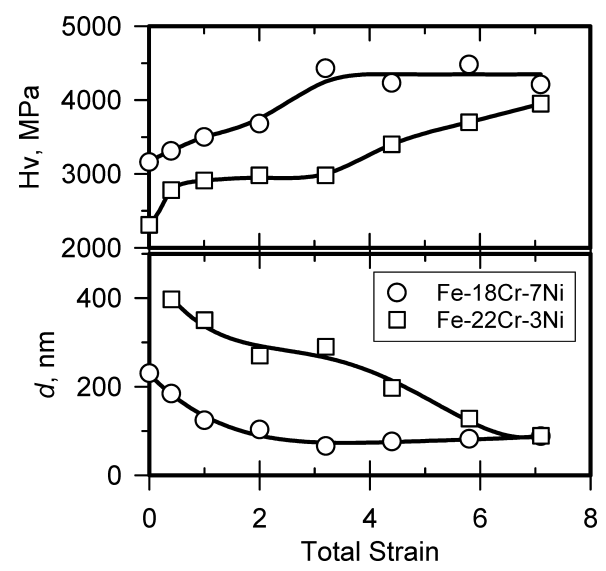

Fig. 13. The change in the hardness and transverse (sub)grain size during cold bar rolling/swaging of $\mathrm{Fe}-22 \mathrm{Cr}-3 \mathrm{Ni}$ and $\mathrm{Fe}-18 \mathrm{Cr}-7 \mathrm{Ni}$.

teristic flat-type misorientation distribution with almost equal fractions of various misorientations is evolved after cold working to rather large total strain of above 4 . In contrast, the samples with original martensitic structure are characterized by two maximums corresponding to low- and high-angle boundaries. The both peaks quickly weaken upon processing to total strain of about 3, leading to the flat-type distribution, which is similar to those developed in coarse grained samples at remarkably larger strains. Similar acceleration of continuous dynamic recrystallization was observed in austenitic stainless steel during warm multiple deformation, when the fraction of strain-induced high-angle grain boundaries in the samples with initial fine grained microstructure exceeded 0.6 after processing to relatively small total strains of about $1 .{ }^{47)}$

The strain hardening and the reduction in the transverse (sub)grain size in the samples with different starting microstructures during large strain cold working is shown in Fig. 13. Comparing to the samples with coarse initial grains, the hardness of the samples with initial martensitic structure rapidly increases to its saturation level after processing to relatively small total strain of around 3 . Corre-

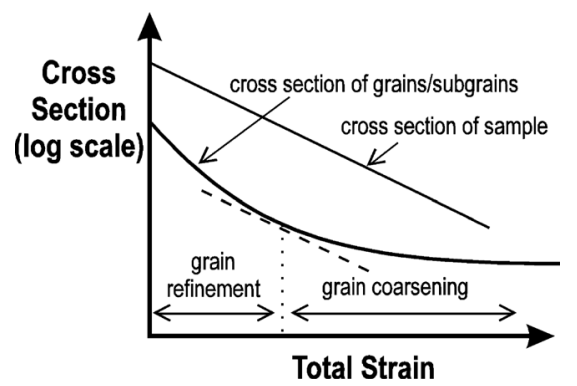

Fig. 14. Schematic illustration for variations of cross sections of grains/subgrains and whole sample with total strain upon unidirectional deformation.

spondingly, the transverse (sub)grain size approaches the minimal value at the same strains, and then does not change significantly during subsequent deformation. Thus, the development of the continuous dynamic recrystallization in the originally martensitic structure resulted in a steadystate-like deformation behavior at strains of above 3 even during cold working.

\section{Apparent Steady-state Deformation}

The strain effect on the reduction in cross section of grains/subgrains and whole sample during unidirectional deformation is schematically illustrated by Fig. 14. The cross section of the sample reduces with total strain as $\exp (-0.5 \varepsilon)$ during bar rolling/swaging or $\exp (-\varepsilon)$ for plane rolling and, therefore, can be represented by a linear plot in semi-logarithmic coordinates. In the range of relatively small to medium total strains, the cross section of grains/subgrains decreases much faster than that of the whole sample. This is the region of grain refinement, when the plastic working results in the development of new (sub)boundaries leading to rapid reduction in the average transverse size of deformation grains and subgrains. In other words, the deformation is accompanied by increasing the density of plane defects of crystal lattice per a grain, i.e. defect accumulation. On the other hand, the number of grains/subgrains in the cross section of sample decreases in the range of large total strains. In spite of a little decrease in the transverse grain/subgrain size with increasing the total strain, the large total strains can be considered as the region of grain coarsening, i.e. defect annihilation becomes dominant process, leading to the apparent steady-state deformation behavior.

The structural response leading to apparent steady-state deformation during unidirectional cold working is not clearly understood. There might be three possible mechanisms keeping the average value of cross section of elongated grains/subgrains as a constant during processing: (i) a shrinkage of individual grains/subgrains, ${ }^{24)}$ (ii) a strain localization by microshearing, ${ }^{25)}$ and (iii) a subgrain coalescence by the plastic hinge mechanism. ${ }^{35)}$ Recovery controlled local migration of strain-induced (sub)boundaries bearing high internal stresses may lead to serration of (sub)boundaries in ribbon-like deformation structures. Therefore, adjacent (sub)boundaries of highly elongated grains/subgrains may pinch off at sufficiently large reductions in cross section of samples, leading the ribbon-like 


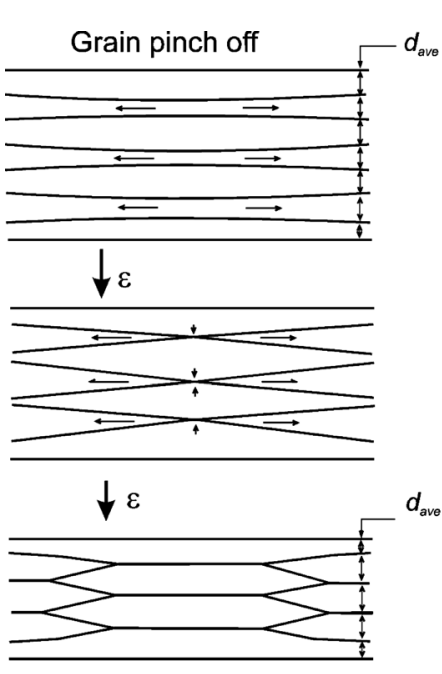

(a)

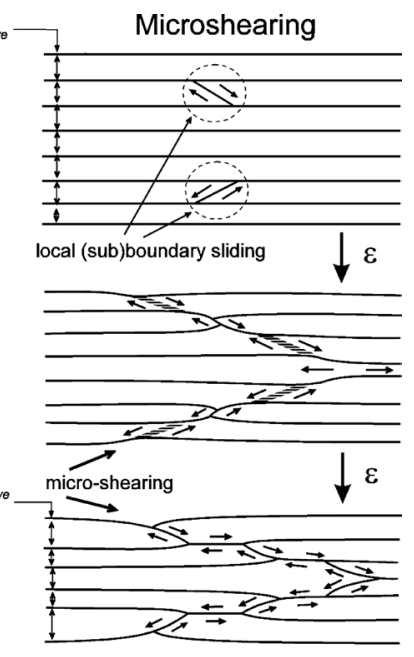

(b)
Fig. 15. Schematic drawing of the structural mechanisms operating upon large strain unidirectional deformation: (a) the shrinkage of elongated grains/subgrains by pinching off their (sub)boundaries and (b) the micro-shears in the ribbon-like structures.

(sub)grains to rupture (Fig. 15(a)). Also, the sequential microshearing resulting in a strain localization on mesoscale level was considered as a possible deformation mechanism operating at large strains in ribbon-like deformation structures (Fig. 15(b)). The microshearing can provide the required dimensional change of whole samples, while the average cross section of grains/subgrains does not reduce. Probably, all these mechanisms operate simultaneously during large strain unidirectional working. The mean contribution (or importance) of each mechanism should be further explored in more detail.

\section{Summary}

The mechanism of submicrocrystalline microstructure evolution in ferritic stainless steels during large strain coldworking can be considered as a kind of continuous dynamic recrystallization. The new ultrafine grains develop at large strains as a result of gradual increase in the misorientations among deformation subgrains. After rapid reduction at an early deformation, the transverse (sub)grain size smoothly approaches its final value of 100-200 nm, which depends on alloying extent. The increase in average subboundary misorientation during deformation can be related to the strain as $\Delta \theta \sim 5^{\circ} \varepsilon$. After cold rolling/swaging to total strain of 4 , the average misorientation between deformation (sub)grains is about $20^{\circ}$, and the fraction of high-angle grain boundaries is 0.5 . Therefore, the level of cold strains about 4 can be considered as a large enough one for almost full development of strain-induced ultrafine grained structure.

The development of submicrocrystalline structures has a little dependency on processing method. The unidirectional deformation by rolling/swaging and the multidirectional processing by $3 \mathrm{D}$ forging are characterised by almost the same strain dependencies for the reduction in the transverse grain and subgrain sizes, which approach $0.3 \mu \mathrm{m}$ and $200 \mathrm{~nm}$ at total strains of around 7. Irrespective of process- ing method, the fraction of high-angle grain boundaries follows the same linear dependency on total strain in the range of $0<\varepsilon<3$, and then saturates at about 0.8 and 0.6 during the unidirectional and multidirectional deformations, respectively.

The steel with initial fine-grained structure demonstrates faster kinetics of continuous dynamic recrystallization during cold-working, i.e. decreasing the transverse (sub)grain size, increasing the fraction of high-angle grain boundaries, than the samples with initial coarse grained microstructure. It is possible to attain an apparent steady-state deformation behaviour during unidirectional cold working by using the samples with initial fine grained martensitic structure.

\section{Acknowledgements}

This study was supported by the Special Coordination Funds of the Ministry of Education, Culture, Sports, Science, and Technology of the Japanese Government. The authors are grateful for useful discussions with Prof. T. Sakai, Prof. H. Miura (The University of Electro-Communications, Tokyo), and Prof. Y. Kimura, Prof. Y. Mishima (Tokyo Institute of Technology, Yokohama).

\section{REFERENCES}

1) K. G. Farkhutdinov, R. G. Zaripova and N. A. Breikina: Mater. Sci. Eng. A, $\mathbf{A 1 7 4}$ (1994), 217.

2) C. Suryanarayana: Int. Mater. Rev., 40 (1995), 41.

3) F. J. Humphreys, P. B. Prangnell, J. R. Bowen, A. Gholinia and C. Harris: Philos. Trans. R. Soc. (London), 357 (1999), 1663.

4) S. Lee, P. B. Berbon, M. Furukawa, Z. Horita, M. Nemoto, N. K. Tsenev, R. Z. Valiev and T. G. Langdon: Mater. Sci. Eng. A, $\mathbf{A 2 7 2}$ (1999), 63.

5) R. Z. Valiev, R. K. Islamgaliev and I. V. Alexandrov: Prog. Mater. Sci., 45 (2000), 103.

6) D. G. Morris: Science of Metastable and Nanocrystalline Alloys, ed. by A. R. Dinesen et al., Riso National Laboratory, Roskilde, Denmark, (2001), 89.

7) Y. Wang, M. Chen, F. Zhou and E. Ma: Nature (London), 419 (2002), 912.

8) A. Vinogradov, V. Patlan, Y. Suzuki, K. Kitagawa and V. I. Kopylov: Acta Mater, 50 (2002), 1639.

9) V. V. Stolyarov, R. Z. Valiev and Y. T. Zhu: Appl. Phys. Lett., 88 (2006), Art. No. 041905.

10) H. J. McQueen and J. J. Jonas: Treatise on Materials Science and Technology, ed. by R. J. Arsenoult, Academic Press, New York, NY, (1975), 393.

11) T. Maki, T. Akasaka, K. Ohkuno and I. Tamura: Trans. Iron Steel Inst. Jpn., 22 (1982), 253.

12) T. Sakai and J. J. Jonas: Acta Metall., 32 (1984), 189.

13) F. J. Humphreys and M. Hatherly: Recrystallization and Related Annealing Phenomena, Pergamon Press, Oxford, (1996), 363.

14) A. Belyakov, H. Miura and T. Sakai: Mater. Sci. Eng. A, $\mathbf{A 2 5 5}$ (1998), 139.

15) V. M. Segal, V. I. Reznikov, A. E. Drobyshevskiy and V. I. Kopylov: Russ. Metall., 1 (1981), 115.

16) I. Saunders and J. Nutting: Met. Sci., 18 (1984), 571.

17) J. Richert and M. Richert: Aluminium, 62 (1986), 604.

18) Y. Saito, N. Tsuji, H. Utsunomiya, T. Sakai and R. G. Hong: Scr. Mater, 39 (1998), 1221.

19) S. Takaki, T. Tsuchiyama, K. Nakashima, H. Hidaka, K. Kawasaki and Y. Futamura: Met. Mater. Int., 10 (2004), 533.

20) A. Belyakov, T. Sakai and H. Miura: Mater. Trans., JIM, 41 (2000), 476.

21) A. Belyakov, T. Sakai, H. Miura and K. Tsuzaki: Philos. Mag. A, 81 (2001), 2629.

22) Yu. Ivanisenko, W. Lojkowski, R. Z. Valiev and H.-J. Fecht: Acta Mater, 51 (2003), 5555.

23) C. Xu, M. Furukawa, Z. Horita and T. G. Langdon: Mater. Sci. Eng. 
ISIJ International, Vol. 48 (2008), No. 8

A, $\mathbf{A 3 9 8}$ (2005), 66.

24) A. Belyakov, Y. Kimura, Y. Adachi and K. Tsuzaki: Mater. Trans., 45 (2004), 2812

25) A. Belyakov, Y. Kimura and K. Tsuzaki: Acta Mater., 54 (2006), 2521.

26) A. Belyakov, K. Tsuzaki, Y. Kimura, Y. Kimura and Y. Mishima: Mater. Sci. Eng. A, A456 (2007), 323.

27) J. Gill Sevillano, P. Van Houtte and E. Aernoudt: Prog. Mat. Sci., 25 (1981), 69.

28) N. Hansen: Metall. Trans. A, 16A (1985), 2167.

29) S. Dymek and M. Blicharski: Z. Metallkd., 76 (1985), 777

30) B. Bay, N. Hansen, D. A. Hughes and D. Kuhlmann-Wilsdorf: Acta Metall. Mater., 40 (1992), 205

31) D. Kuhlmann-Wilsdorf and N. Hansen: Scr. Metall. Mater, 25 (1991), 1557.

32) D. A. Hughes and N. Hansen: Acta Mater, 45 (1997), 3871.

33) A. S. Argon and P. Haasen: Acta Metall. Mater., 41 (1993), 3289.

34) J. D. Embury, A. S. Keh and R. M. Fisher: Trans. AIME, 236 (1966), 1252.

35) G. Langford and M. Cohen: Trans. ASM, 62 (1969), 623.

36) A. Ball, F. P. Bullen, F. Henderson and H. L. Wain: Philos. Mag., 21 (1970), 701.
37) A. Belyakov, Y. Kimura, Y. Adachi and K. Tsuzaki: Evolution of Deformation Microstructures in 3D, ed. by C. Gundlach et al., Riso National Laboratory, Roskilde, Denmark, (2004), 239.

38) A. Belyakov, R. Kaibyshev and T. Sakai: Metall. Mater. Trans. A, 29A (1998), 161

39) A. Belyakov, W. Gao, H. Miura and T. Sakai: Metall. Mater. Trans. A, 29A (1998), 2957.

40) T. Sakai: Ultrafine Grained Materials IV, ed. by Y. T. Zhu et al., TMS, Warrendale, PA, (2006), 73.

41) K. Tsuzaki, X. Huang and T. Maki: Acta Mater, 44 (1996), 4491.

42) Q. Liu, X. Huang, M. Yao and J. Yang: Acta Mater., 40 (1992), 1753.

$43)$ D. Hull and D. J. Bacon: Introduction to Dislocations, ButterworthHeinemann, Oxford, UK, (1984), 67.

44) A. Belyakov, T. Sakai, H. Miura and R. Kaibyshev: Scr. Mater, 42 (2000), 319.

45) A. Belyakov, T. Sakai, H. Miura and R. Kaibyshev: Philos. Mag. Lett., 80 (2000), 711.

46) A. Belyakov, Y. Sakai, T. Hara, Y. Kimura and K. Tsuzaki: Scr. Mater, 45 (2001), 1213.

47) A. Belyakov, K. Tsuzaki, H. Miura and T. Sakai: Acta Mater, 51 (2003), 847. 